\title{
Extended Erratum to \\ A parabolic flow toward solutions of the optimal transportation problem on domains with boundary
}

(J. reine angew. Math. 672 (2012), 127-160)

By Jun Kitagawa at East Lansing

The author was notified by Neil Trudinger (who the author would like to thank, both for pointing out the original error, and for suggesting adapting the construction below to correct this error) that the proof of the interior $C^{2}$ estimate [3, Theorem 10.1] is incorrect. A previous erratum [4] was issued in which the aforementioned error was not completely corrected, this is rectified in the current erratum. In the original proof, there is a missing term of the form $2 a u_{l} w^{i j} D_{x_{l}} A_{i j}$ in the second displayed block of inequalities on page 148, which can only be bounded from below by a term of the form $-C_{1} a \operatorname{tr}\left(w^{i j}\right)$ for some constant $C_{1}>0$. This changes the inequality in the middle of page 149 to

$$
C_{a}+\left(C+C_{1} a\right) \operatorname{tr}\left(w^{i j}\right) \geq(2 a-C) \operatorname{tr}\left(w_{i j}\right),
$$

hence (after dividing through by $2 a-C$ ), the factor in front of the term $\operatorname{tr}\left(w^{i j}\right)$ cannot be made small by taking $a$ large which is a crucial ingredient in the estimate.

We will now rectify this error by using a barrier developed by Jiang and Trudinger in [1] for the elliptic case. Recall that the linearized operator $L$ in [3, Theorem 10.1] is defined by

$$
L \phi=-\dot{\phi}+w^{i j}(x, t)\left(\phi_{i j}-D_{p_{k}} A_{i j}(x, \nabla u(x, t)) \phi_{k}\right)-D_{p_{k}} \tilde{B}(x, \nabla u(x, t)) \phi_{k},
$$

where $u(x, t)$ is the solution of the parabolic flow and $w^{i j}(x, t)$ are the entries of the inverse matrix of $w_{i j}(x, t):=u_{i j}(x, t)-A_{i j}(x, \nabla u(x, t))$. We will find a function $\psi$ satisfying

$$
L \psi \geq C_{2} \operatorname{tr}\left(w^{i j}\right)-C_{3}
$$

with $0 \leq \psi(x, t) \leq C_{4}$ for some constants $C_{2}, C_{3}, C_{4}>0$ independent of the maximum time of existence $t_{\max }$ for $u$. If this is possible, by redefining the auxiliary function used in [3, Theorem 10.1] as

$$
v(x, t, \xi):=\log w_{\xi \xi}(x, t)+a|\nabla u(x, t)|^{2}+\frac{C_{1}}{C_{2}} a \psi(x, t),
$$

(c) BY (C) 2021 Jun Kitagawa, published by De Gruyter. This work is licensed under the Creative Commons Attribution 4.0 International License. 
where $C_{1}$ is the constant in inequality (1), and then evaluating $L v$ at an interior maximum $\left(x_{0}, t_{0}, \xi_{0}\right) \in \Omega \times\left[0, t_{\max }\right) \times \mathbb{S}^{n-1}$, we can recover the correct estimate of the form

$$
C_{a}+C \operatorname{tr}\left(w^{i j}\right) \geq(2 a-C) \operatorname{tr}\left(w_{i j}\right)
$$

on page 149 . The proof can then be continued as written to obtain the inequality

$$
\operatorname{tr} w_{i j}\left(x_{0}, t_{0}\right) \leq \tilde{C}
$$

for some uniform constant $\tilde{C}$. Then, for arbitrary $(x, t, \xi) \in \Omega \times\left[0, t_{\max }\right) \times \mathbb{S}^{n-1}$, we have

$$
w_{\xi \xi}(x, t) \leq e^{v(x, t, \xi)} \leq e^{v\left(x_{0}, t_{0}, \xi_{0}\right)}=e^{a\left|\nabla u\left(x_{0}, t_{0}\right)\right|^{2}+\frac{C_{1}}{C_{2}} a \psi\left(x_{0}, t_{0}\right)} w_{\xi_{0} \xi_{0}}\left(x_{0}, t_{0}\right) \leq C
$$

for a $C>0$ independent of $t_{\max }$, yielding the desired interior $C^{2}$ estimate.

To show such a $\psi$ exists, we rely on a barrier construction presented in [1]; let us also note that a simpler proof of this construction can also be found in the discussion leading up to [2, Lemma 2.1]. First let

$$
U(t):=\frac{1}{|\Omega|} \int_{\Omega} u(z, t) d z
$$

since $\dot{u}$ is uniformly bounded by [3, Theorem 5.1], we see $U \in C^{1}\left(\left[0, t_{\max }\right)\right)$. Now fix an element $(x, t) \in \Omega \times\left[0, t_{\max }\right)$ and write $y:=Y(x, \nabla u(x, t))$; then for any $z \in \Omega$, if we let

$$
x_{z}(s):=X\left((1-s) \nabla_{y} c(x, y)+s \nabla_{y} c(z, y), y\right),
$$

we have the bound

$$
\begin{aligned}
|U(t)-u(x, t)| & \leq \frac{1}{|\Omega|} \int_{\Omega} \int_{0}^{1}\left|\left\langle\nabla u\left(x_{z}(s), t\right), \dot{x}_{z}(s)\right\rangle\right| d s d z \\
& \leq \frac{2 \sup _{\Omega \times\left[0, t_{\max }\right)}|\nabla u| \sup \left\|\left(D_{x, y}^{2} c\right)^{-1}\right\| \sup \left|\nabla_{y} c\right|}{|\Omega|}
\end{aligned}
$$

which is bounded independent of $t_{\max }$ by [3, Theorem 6.1]. Now note that in the notation of [1], for the choice of $g(x, y, z)=c(x, y)-z$ the conditions A1, A2, A ${ }^{*}, \mathrm{~A} 3 \mathrm{w}, \mathrm{A} 4 \mathrm{w}$, and all domain convexity conditions in [1] will be satisfied under the current hypotheses. Next, in the construction of the function $\bar{u}$ in [1, Lemma 2.1], in this optimal transport case it is easy to see that any derivative of $\bar{u}$ is independent of the choice of $z_{0}$, while the matrix valued functions $A$ and $A^{*}$ are independent of the scalar variable $z$, hence the constant $a_{0}$ is independent of the value of the scalar $z_{0}$. In turn, we see the constant $a_{1}=n \log \frac{a_{0}}{2}$ appearing in [1, Lemma 2.2] is also independent of the choice of $z_{0}$. Thus by (3) we can translate by a constant to assume that $0 \leq \bar{u}(x)+U(t)-u(x, t)$, and we define

$$
\psi(x, t):=e^{K(\bar{u}(x)+U(t)-u(x, t))},
$$

by (3) we see $\psi$ also has an upper bound independent of $t_{\max }$.

Let us write $\tilde{L} \phi:=w^{i j}(x, t)\left(\phi_{i j}-D_{p_{k}} A_{i j}(x, \nabla u(x, t)) \phi_{k}\right)$. Then examining the final displayed equation in the proof of [1, Lemma 2.2] reveals that

$$
\tilde{L} e^{K(\bar{u}(x)-u(x, t))} \geq e^{K(\bar{u}(x)-u(x, t))}\left[C_{2} \operatorname{tr} w^{i j}-\tilde{C}_{3}\right]
$$

for some $C_{2}, \tilde{C}_{3}>0$ independent of $t_{\max }$ (the only difference between the use of $u(x, t)$ versus $u(x)$ is that the term $F[u]$ in the proof of [1, Lemma 2.2] will include a term $-\dot{u}$, which is 
bounded by [3, Theorem 8.1]). Then again using [3, Theorems 6.1 and 8.1], and the upper and lower bounds on $\psi$, we calculate

$$
\begin{aligned}
L \psi & =e^{K U(t)} \tilde{L} e^{K(\bar{u}(x)-u(x, t))}-K \psi(x, t) D_{p_{k}} \tilde{B}\left(\bar{u}_{k}(x)-u_{k}(x, t)\right) \\
& -K \psi(x, t)(\dot{U}(t)-\dot{u}(x, t)) \\
& \geq C_{2} \psi(x, t) \operatorname{tr} w^{i j}-\psi(x, t) \tilde{C}_{3}-K \sup |\psi|\left(\sup \left|D_{p_{k}} \tilde{B}\right|(|\nabla \bar{u}|+|\nabla u|)+2 \sup |\dot{u}|\right) \\
& \geq C_{2} \operatorname{tr} w^{i j}-C_{3} .
\end{aligned}
$$

Thus $\psi$ has the desired property.

\section{References}

[1] F. Jiang and N.S. Trudinger, On Pogorelov estimates in optimal transportation and geometric optics, Bull. Math. Sci. 4 (2014), no. 3, 407-431.

[2] F. Jiang and N.S. Trudinger, Oblique boundary value problems for augmented Hessian equations II, Nonlinear Anal. 154 (2017), 148-173.

[3] J. Kitagawa, A parabolic flow toward solutions of the optimal transportation problem on domains with boundary, J. reine angew. Math. 672 (2012), 127-160.

[4] J. Kitagawa, Erratum to A parabolic flow toward solutions of the optimal transportation problem on domains with boundary (J. reine angew. Math. 672 (2012), 127-160), J. reine angew. Math. 760 (2020), 165-167.

Jun Kitagawa, Department of Mathematics, Michigan State University, East Lansing, MI 48824, USA

https://orcid.org/0000-0002-6145-6664

e-mail: kitagawa@math.msu.edu

Eingegangen 2. August 2021, in revidierter Fassung 8. September 2021 